\title{
Total Ankylosis by Heterotopic Ossification in an Adolescent Anterior Trans-olecranon Fracture Dislocation: A Case Report
}

\author{
Beom-Soo Kim, Kwang-Soon Song, Ki-Cheor Bae, Si-Wook Lee, Sang-Hyun Um, Chul-Hyun Cho \\ Department of Orthopedic Surgery, Dongsan Medical Center, Keimyung University School of Medicine, Daegu, Korea
}

\begin{abstract}
The incidence of heterotopic ossification in adolescents appears to be lower than in adults. There exist very few reports of heterotopic ossification with total bony ankylosis in child or adolescent populations. We describe a case of total bony ankylosis of the elbow secondary to heterotopic ossification, in a 14-year-old female. Total ankylosis of the elbow at 45 degrees of flexion was noted 6 months postsurgery, and complete surgical excision of the heterotopic mass was performed. After an additional one-time dose of radiation therapy and nonsteroidal anti-inflammatory drug medication, full range of motion was obtained without any recurrence or other complications, up to the last follow-up of 30 months.
\end{abstract}

(Clin Shoulder Elbow 2019;22(3):154-158)

Key Words: Adolescent; Heterotopic ossification; Elbow; Ankylosis; Bone fractures

Heterotopic ossification ( $\mathrm{HO}$ ) of the upper limb is an uncommon post-traumatic complication, and is even rarer among children. ${ }^{1)}$ The occurrence of $\mathrm{HO}$ around the elbow joint results in fixed deformities and complete limitation of motion. ${ }^{2,3)}$ Surgical excision of $\mathrm{HO}$ is subsequently required for patients afflicted with limitated motion, and complete surgical excision can achieve a significant improvement of elbow functions. ${ }^{4,5)}$

Total ankylosis of the elbow joint due to $\mathrm{HO}$ is not common, and very little is known about $\mathrm{HO}$ treatment in children or adolescents. To the best of our knowledge, surgical excision of $\mathrm{HO}$ around the elbow joint in an adolescent has not been performed previously. We describe a case of total ankylosis of the elbow joint by $\mathrm{HO}$ in a 14-year-old female with anterior transolecranon fracture dislocation, who was successfully treated by complete surgical excision of the $\mathrm{HO}$.

\section{Case Report}

A 14-year-old right-handed female fell from a 10th floor apartment, and presented to our emergency department with multiple trauma. She had no history of any previous illness or medication before the injury. She complained of pain in the abdomen, right elbow, pelvic area, and low back area. She underwent iliac artery embolization and exploratory laparotomy to repair the iliac artery and a liver laceration at another hospital, and was subsequently transferred to our emergency center for additional surgical intervention involving the pelvic bone, spine and anterior trans-olecranon fracture dislocation (Fig. 1). Fortunately, computed tomography (CT) confirmed that she had no brain injury and no systematic neurologic symptoms, although there was a chance fracture of the T10 vertebra. She was subjected twice to a closed reduction of the injured elbow joint, once at the first hospital, and then in our emergency room. According to the Mayo classification, the olecranon fracture was diagnosed as type IIIB. Sixteen days after the initial trauma, the anterior trans-olecranon fracture dislocation of the right elbow joint was treated by open reduction and internal fixation by applying a pre-contoured olecranon plate (Zimmer, Warsaw, IN,

Received March 6, 2019. Revised April 9, 2019. Accepted May 18, 2019.

Correspondence to: Chul-Hyun Cho

Department of Orthopedic Surgery, Dongsan Medical Center, Keimyung University School of Medicine, 56 Dalseong-ro, Jung-gu, Daegu 41931, Korea

Tel: +82-53-250-7729, Fax: +82-53-250-7205, E-mail: oscho5362@dsmc.or.kr, ORCID: https://orcid.org/0000-0003-0252-8741

IRB approval: Keimyung University Dongsan Medical Center (DSMC 2019-03-024).

Financial support: None. Conflict of interests: None. 

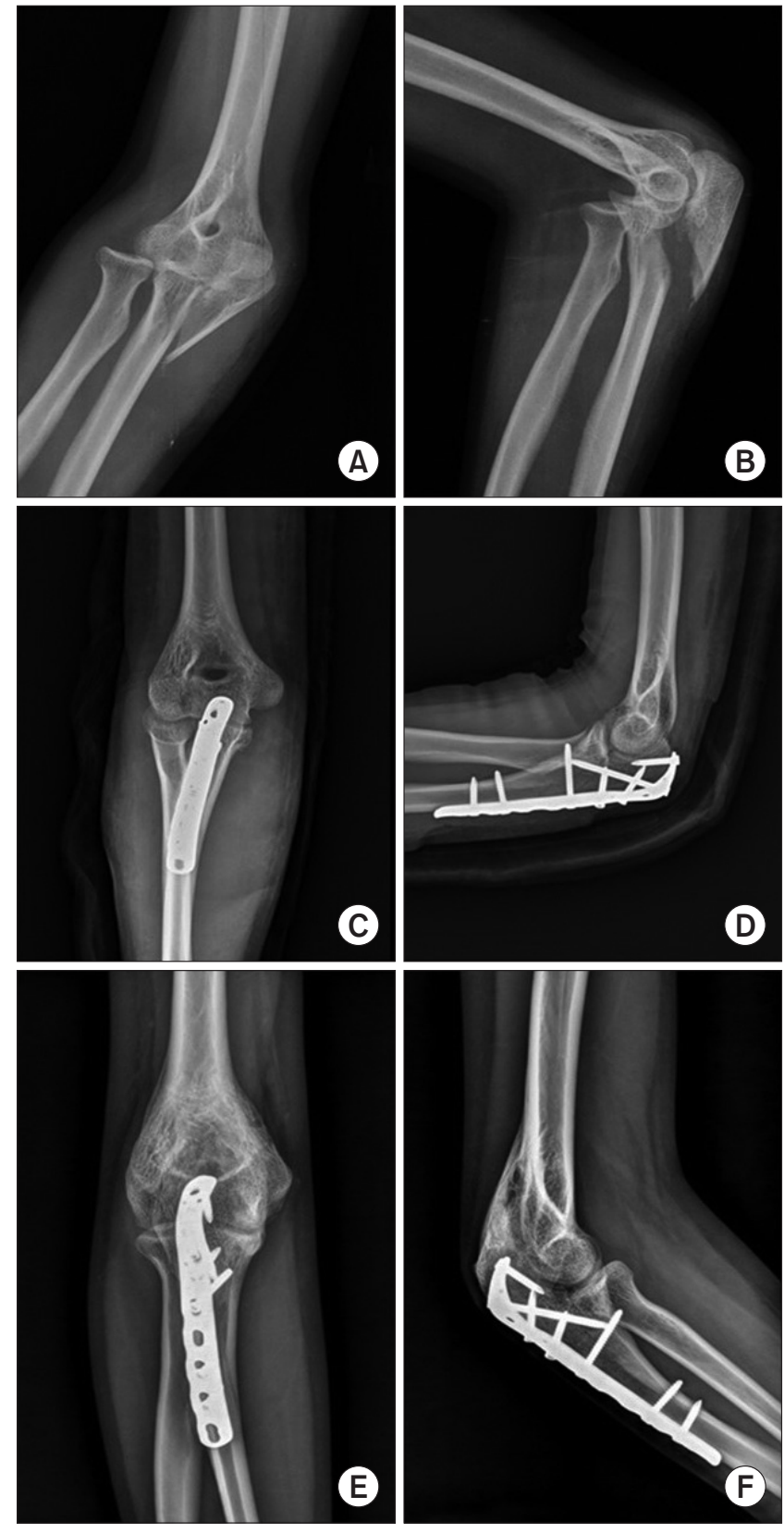

Fig. 1. (A, B) Anteroposterior and lateral elbow radiographs at initial trauma show ulna olecranon fracture with anterolateral elbow dislocation. (C, D) Open reduction and internal fixation using olecranon locking plate was done 16 days after the initial trauma, and are presented as anteroposterior and lateral radiographs. (E, F) Anteroposterior and lateral radiographs of the affected elbow reveal a large bony mass connecting the distal humerus to the ulna at 6 months of index operation.

USA) using the posterior approach incised along the triceps, followed by application of a long arm splint (Fig. 1).

After surgery, the elbow was immobilized in a long arm splint at 90 degrees of flexion and neutral rotation. Starting from the 3rd postoperative day, the patient was allowed gentle exercise of the right elbow joint, but developed aggravated pain during rehabilitation at 2 weeks post-surgery. The $\mathrm{HO}$ was first noted in radiographs, at 3 weeks. At 10 weeks post-surgery, she had increased difficulty in moving her right elbow joint, and had reduced range of motion. The size of $\mathrm{HO}$ had progressed and was clearly revealed in radiographs. Physical examination revealed total ankylosis of the right elbow at 45 degrees of flexion, at post-operative 6 months. However, since the radioulnar joint was intact, pronation and supination of the forearm showed the full range of joint motion. Standard anteroposterior and lateral radiographs of the affected elbow revealed an unusually large mass connecting the distal humerus to the ulna (Fig. 1, 2). CT scan images revealed the progress of mineralization from the outer margins towards the center area (Fig. 3). After 6 months maturation of the heterotopic mass, surgical excision was carried out using the posterior approach through the previous incision scar. Skin, subcutaneous tissue, and deep fascia were incised in line with the skin incision. The ulnar nerve was identified and retracted. The extent of the mass was exposed adequately and was excised completely from the distal humerus and ulna (Fig. 2). Intraoperative elbow range of motion flexion 0 to 140 degree was checked.

Postoperative radiographs showed no trace of HO (Fig. 4). One day after the operation, a 1-time dose of radiation therapy (800 cGy) was provided to prevent recurrence. Four days after surgery, the elbow range of motion exercise was started, as tolerated.

At 30 months after surgery, patient had no pain in the elbow; radiographs revealed no further development of the $\mathrm{HO}$, and a residual flexion deformity of 10 degrees with flexion up to 145 degrees, and supination and pronation of the forearm were achieved with full range of motion (Fig. 4, 5). The patient was permitted to return to play, without any restrictions.

The Patient was given an opportunity to review the manuscript and consented to its publication.

\section{Discussion}

In the current case, the $\mathrm{HO}$ around the elbow was especially problematic for many reasons. Since the neurovascular structure lies within the elbow joint, this increased the possibility of complications. Second, the ankylotic tendency of the elbow joint to occur after injury further complicates the treatment of heterotopic ossification in the elbow joint. ${ }^{2)}$

Complete ankylosis of the elbow secondary to $\mathrm{HO}$ results in severe disability. More than 20\% patients who develop $\mathrm{HO}$ have clinically limited motion in the form of decreased arc of the flexion-extension to under 100 degrees. In our case, the $\mathrm{HO}$ was located on the posteromedial side, and the elbow range of motion was assessed as 30 to 60 degrees at post-operative 4 weeks, which gradually progressed to total ankylosis of the right elbow at 45 degrees of flexion. Restoration of elbow motion with complete ankylosis can be difficult. ${ }^{6}$ During surgery, 

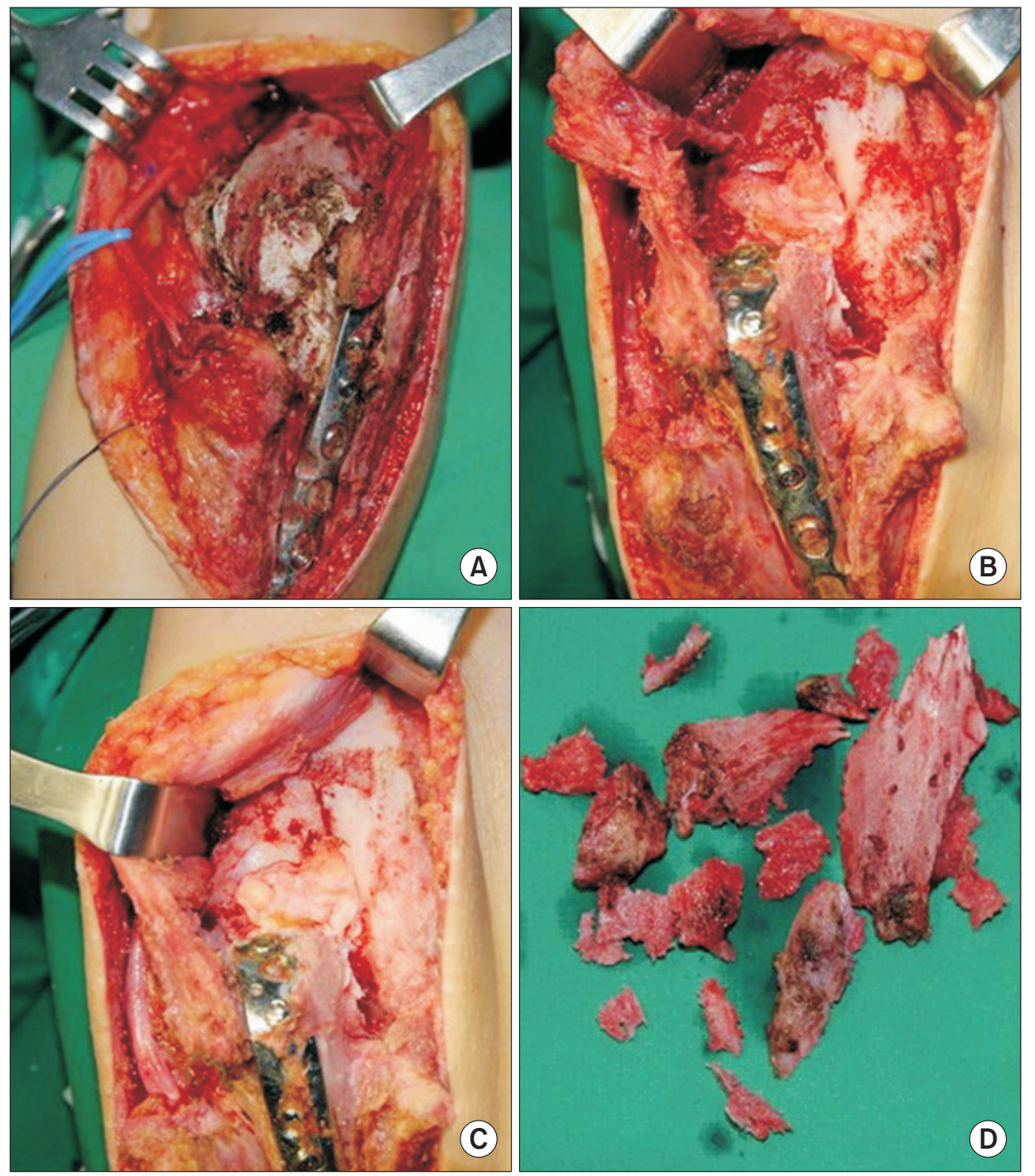

Fig. 2. (A) An intraoperative view of the untreated heterotopic ossification of the posterior elbow joint shows a neurovascular bundle, which was identified and retracted. (B) An intraoperative view of the posterior elbow joint. (C, D) Elbow joint after excision of the mass of bone.

Fig. 3. (A, B) Images of computed tomography scan reveal fully matured mineralized cortical bone connecting the distal humerus to the ulna. 


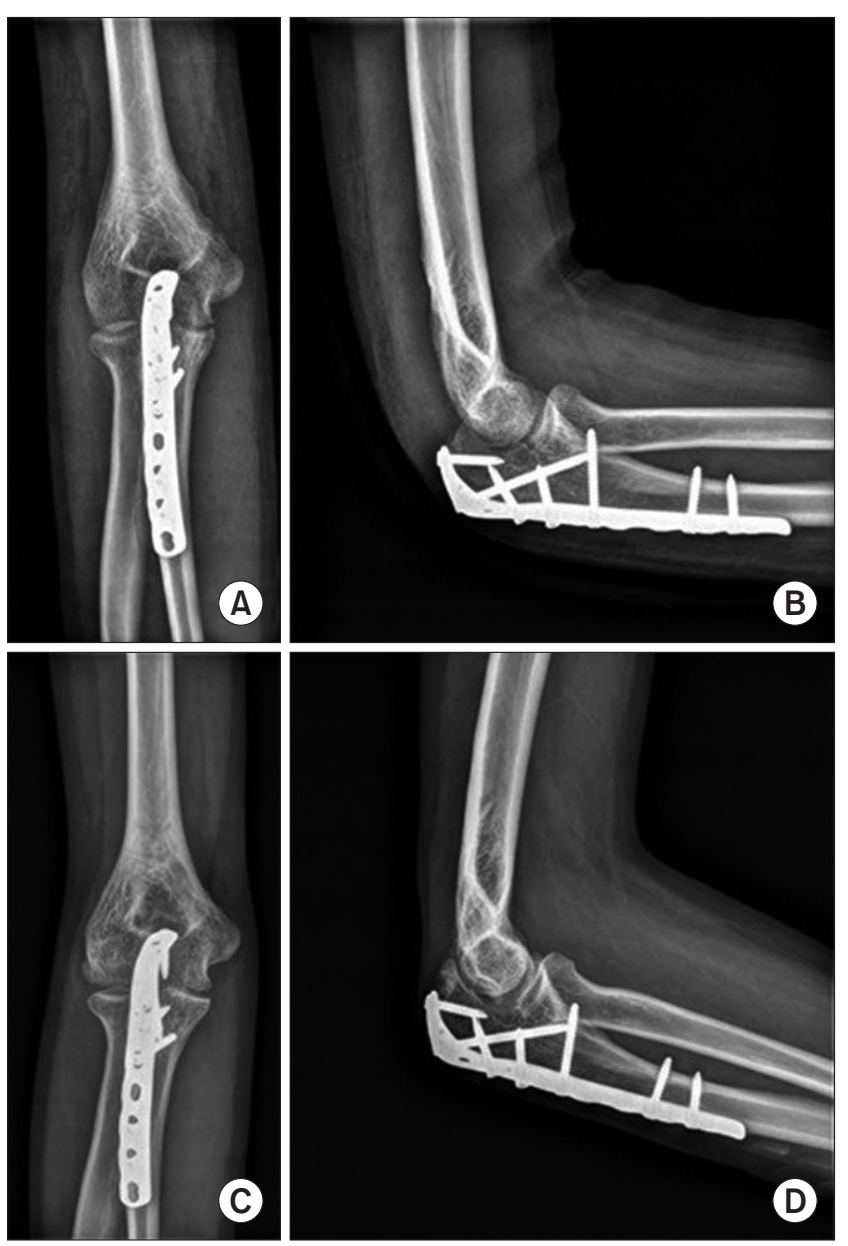

Fig. 4. (A, B) Postoperative anteroposterior and lateral elbow radiographs show complete excision status of a heterotopic ossification. (C, D) Radiographs show no further development of heterotopic ossification of the elbow joint at 30 months after index operation. after resection of $\mathrm{HO}$, the elbow range of motion was found to be 0 to 140 degrees. At the final follow-up, the range of motion checked at 10 to 145 degrees.

Surgical removal of the ectopic bone should be undertaken only for clear functional goals. Excision should be considered for patients in whom elbow motion is severely limited by extensive $\mathrm{HO}$ and other neurologic symptoms of the elbow joint. In the current case, our patient had complains of ulnar nerve symptoms such as tingling sensation and numbness. Since timing surgical excision of the heterotopic bone is important ${ }^{7)}$ and maturation of the $\mathrm{HO}$ was noted on radiographs after 6-month follow-up in our patient, we determined to remove the mass at that instance.

Risk factors of $\mathrm{HO}$ development include neurologic injury, delayed internal fixation, use of bone graft, and the pattern of the fracture. ${ }^{2)}$ Delay in surgery increases the risk for $\mathrm{HO}$, thereby prompting that operative fixation is imperative. ${ }^{8)}$ In our case, internal organ damage had made her vital signs unstable, and we first had to manage life threatening complications. The injured elbow was therefore treated 16 days after the initial trauma, which may have increased the possibility of the developing $\mathrm{HO}$.

Radiation is effective for prophylaxis and recurrence against HO. Radiation may be given at a dose of 700 to 800 cGy in single fraction, administered from 24 hours preoperatively until 48 to 72 hours postoperatively. ${ }^{3)}$ In our case, the patient was given a single dose of 800 cGy 1 day after surgery to prevent recurrence. Furthermore, prophylactic management was administered by prescribing nonsteroidal anti-inflammatory drugs (NSAIDs) for 2 months. NSAIDs prevent $\mathrm{HO}$ by inhibiting the osteogenic differentiation of progenitor cells. ${ }^{9)}$ Radiation and the NSAID therapy are both effective prophylactic treatments for the development of $\mathrm{HO} .{ }^{10)}$ At the last follow-up, no recurrence was observed.
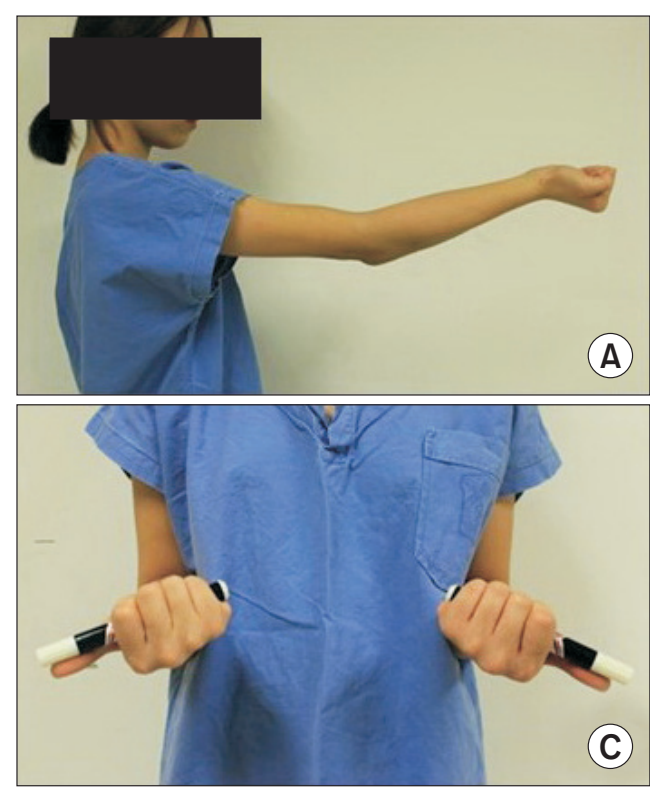

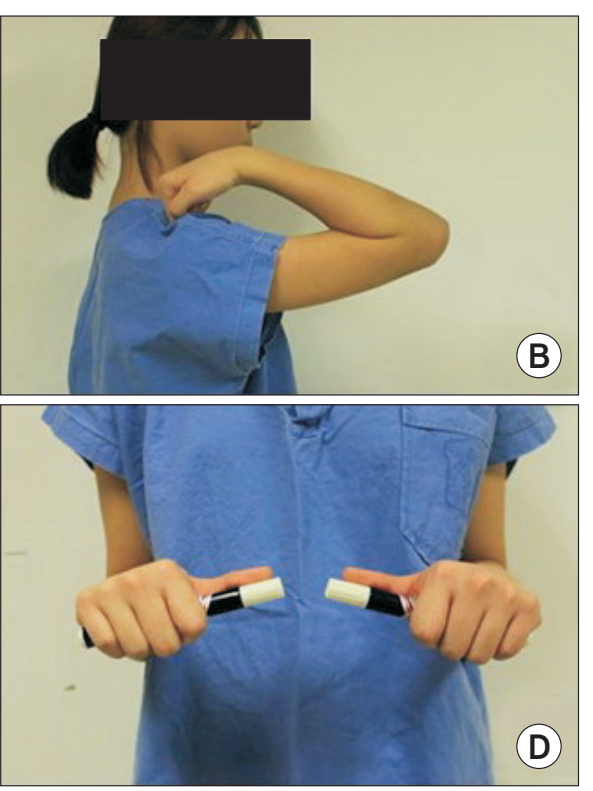

Fig. 5. Clinical photographs of extension and flexion of the elbow joint (A, B), and supination and pronation of the forearm showing functional outcome at 30 months after last surgery (C, D). The Patient was given an opportunity to review the manuscript and consented to its publication. 
In conclusion, surgical excision for a patient who had a fully mature $\mathrm{HO}$ bridging led to significant improvement in the elbow range of motion, ulnar nerve symptoms, and functional results, notwithstanding the subject being an adolescent patient. Complete excision with subsequent postoperative radiation and NSAID therapy helped to prevent recurrence of $\mathrm{HO}$.

\section{References}

1. Kanthimathi B, Udhaya Shankar S, Arun Kumar K, Narayanan VL. Myositis ossificans traumatica causing ankylosis of the elbow. Clin Orthop Surg. 2014;6(4):480-3. doi: 10.4055/ cios.2014.6.4.480.

2. Lee EK, Namdari S, Hosalkar HS, Keenan MA, Baldwin KD. Clinical results of the excision of heterotopic bone around the elbow: a systematic review. J Shoulder Elbow Surg. 2013;22(5):716-22. doi: 10.1016/j.jse.2012.11.020.

3. Susnjar T, Biocić M, Pogorelić Z. Traumatic heterotopic ossification of the elbow in children: a case report. Acta Chir Belg. 2010;110(2):246-9.

4. Ring D, Jupiter JB. Operative release of ankylosis of the elbow due to heterotopic ossification. Surgical technique. J Bone Joint Surg Am. 2004;86 Suppl 1:2-10. doi: 10.2106/00004623200403001-00002.
5. Ring D, Jupiter JB. Operative release of complete ankylosis of the elbow due to heterotopic bone in patients without severe injury of the central nervous system. J Bone Joint Surg Am. 2003;85(5):849-57. doi: 10.2106/00004623-20030500000012.

6. Salazar D, Golz A, Israel H, Marra G. Heterotopic ossification of the elbow treated with surgical resection: risk factors, bony ankylosis, and complications. Clin Orthop Relat Res. 2014;472(7):2269-75. doi: 10.1007/s11999-014-3591-0.

7. Naranje S, Kancherla R, Kannan A, Malhotra R, Sharma $\mathrm{L}$, Sankineani SR. Extraarticular bony ankylosis in a child with supracondylar fracture of humerus. Chin J Traumatol. 2012;15(5):300-2. doi: 10.3760/cma.j.issn.1008-1275.2012. 05.009 .

8. Ranganathan K, Loder S, Agarwal S, et al. Heterotopic ossification: basic-science principles and clinical correlates. J Bone Joint Surg Am. 2015;97(13):1101-11. doi: 10.2106/JBJS.N.01056.

9. Baird EO, Kang QK. Prophylaxis of heterotopic ossification: an updated review. J Orthop Surg Res. 2009;4:12. doi: 10.1186/1749-799X-4-12.

10. Sell S, Willms R, Jany R, et al. The suppression of heterotopic ossifications: radiation versus NSAID therapy: a prospective study. J Arthroplasty. 1998;13(8):854-9. doi: 10.1016/S08835403(98)90189-9. 